\title{
Autonomous di/dt Noise Control Scheme for Margin Aware Operation
}

\author{
Toru Nakura ${ }^{(1)}$, Makoto Ikeda ${ }^{(2)}$ and Kunihiro Asada ${ }^{(2)}$ \\ (1) Dept. of Electronic Engineering, University of Tokyo, Tokyo, Japan. \\ (2) VLSI Design and Education Center (VDEC), University of Tokyo, Tokyo, Japan. \\ \{nakura, ikeda, asada\}@silicon.t.u-tokyo.ac.jp
}

\begin{abstract}
:
This paper demonstrates the first trial of an autonomous and margin aware $d i / d t$ noise control scheme. A $d i / d t$ on the power line is detected by a mutual inductor, the induced voltage is multiplied by Gilbert multiplier and the following low pass filter outputs a DC voltage in proportion to the $d i / d t$ noise. The DC voltage is compared with reference voltages, and the modes of the internal circuit is controlled depending on the comparators output. By using this scheme, the $d i / d t$ noise power can be autonomously controlled to fall within a defined range set by the reference voltages.

We use two operation modes here: all-active and halfactive modes. Our experimental results show that the internal circuit oscillates between the all-active and the halfactive modes, also show that the all/half ratio and the oscillation frequency changes depending on the reference voltages. It proves that our autonomous $d i / d t$ noise control scheme works as being designed.
\end{abstract}

\section{Introduction}

As the process technology advances, the number of transistors on an LSI chip has been increasing and their high speed operations generate more power line noise while the low supply voltage reduces the noise margin. Thus, the power line noise becomes a serious issue for the reliability of the LSI operations. The ground line noise also presents on the substrate[1] because the ground line and the substrate are tied with very low impedance, and hence the power line noise becomes a serious concern for substrate noise on analog-digital mixed signal LSIs as well. The noise on a power line is caused by $d i / d t$ noise and resistive voltage drops due to the parasitic impedance of the power line. As a chip operating frequency becomes higher, the $d i / d t$ noise becomes dominant. An EMI noise is also caused by the $d i / d t$.

It is difficult to predict the amount of the $d i / d t$ noise by a circuit simulation because it requires a huge database of the parasitic impedance, the switching activity and the switching timing, along with very long simulation time. In addition, many recent LSIs have several operating modes. Moreover, a process variation often makes the prediction different from the actual $d i / d t$ noise[2].

This paper proposes an autonomous and margin aware $d i / d t$ noise control scheme. We have proposed an on- chip $d i / d t$ detector[3], and the $d i / d t$ detector can be used for the $d i / d t$ noise control scheme here since the detector is realized on-chip and outputs the $d i / d t$ value in real time. The first trial, as the authors knowledge, of the autonomous and margin aware $d i / d t$ noise control scheme is demonstrated here using $0.15 \mu \mathrm{m}$ 5-ML SOI-CMOS technology.

\section{Circuit Design}

\subsection{Basic Concept}

The block diagram of the autonomous $d i / d t$ noise control scheme is shown in Fig.1. The operation of the internal circuit causes the $d i / d t$ of the power line. The mutual inductor induces the $d i / d t$ proportional voltage between the terminals of the secondary inductor, the induced voltage is squared and the $d i / d t$ noise power waveform is obtained. And the following low pass filter outputs the DC voltage in proportion to the $d i / d t$ noise power. Then the comparators compare the DC voltage with reference voltages $V_{\text {ref } N}$, and the operation mode controller controls the mode of the internal circuit in accordance with the comparator outputs. For example, the modes of the internal circuit include the variations of the clock frequency, effective power supply voltage, threshold voltage, number of the pipeline stage, number of parallel threading and so on. These mode changes result in different $d i / d t$ of the internal circuit and hence the $d i / d t$ noise of the internal circuit can be controlled.

Another advantage of this method is that the allowed $d i / d t$ level can be decided by the reference voltages of the comparators. Thus it is expected that a decided noise margin is autonomously realized by setting the proper reference voltages.

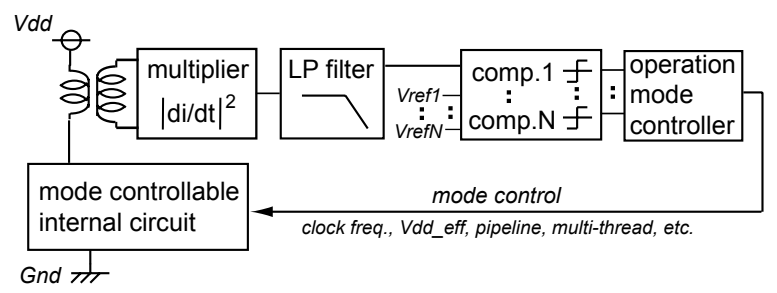

Figure 1: Block diagram of the autonomous $d i / d t$ noise control scheme. 


\subsection{Internal Circuit as Noise Source}

Figure 2 shows our internal circuit as a noise generator. The circuit contains VCO so that we can easily sweep the clock frequency by changing the DC control voltage (Vctrl). The frequency divider generates $101010 \ldots$ signal for the input to the shift resister. The following dividers generates $C L K / 4, C L K / 8$ signals by which the DFF outputs and some inverter chains are disconnected when the divided clock outputs are "Low", and hence the current waveform becomes different in accordance with the divided clock signals. allORhal $f$ signal controls the activation ratio of the circuit. The $C L K / 32$ output is used as a trigger for a oscilloscope, and the $C L K / 2$ output is used to check if the circuit works fine.

Note that the internal circuit has two modes: the all-active and half-active modes.

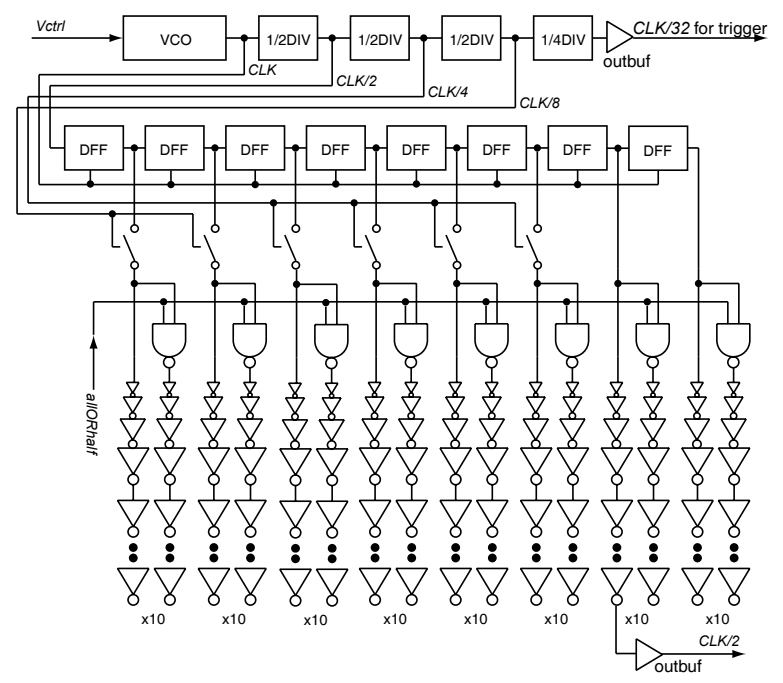

Figure 2: Internal circuit.

\section{$2.3 \mathrm{di} / \mathrm{dt}$ Detector}

The $d i / d t$ detector here is a mutual inductor which induces the $d i / d t$ proportional voltage between the terminals. The mutual inductors here consists of a power supply line and an underlying spiral inductor. The power supply line $L_{1}$ is composed of the top metal layer ML5 with 1 turn, $20 \mu \mathrm{m}$ width. The spiral inductor $L_{2}$ has 20 turns with $2 \mu \mathrm{m}$ width and $2 \mu \mathrm{m}$ spacing using ML3. The outside diameter of the both inductors are $150 \mu \mathrm{m} \times 150 \mu \mathrm{m}$, as shown in Fig.3.

The resistors $R_{b}$ are used to keep the DC bias voltage as half-Vdd for the following input. The resistor is formed using gate-poly without silicide and the resistance is $10 \mathrm{k} \Omega$ which is big enough to be considered open for AC signal.

\section{4 di/dt Multiplier and Low Pass Filter}

A Gilbert multiplier[4] is used for obtaining the square of the $d i / d t$ signal, which converts the $d i / d t$ proportional voltage into the $d i / d t$ noise power waveform. The following low pass filter is composed of a resistor and a capacitor. The schematic is shown in Fig.4.

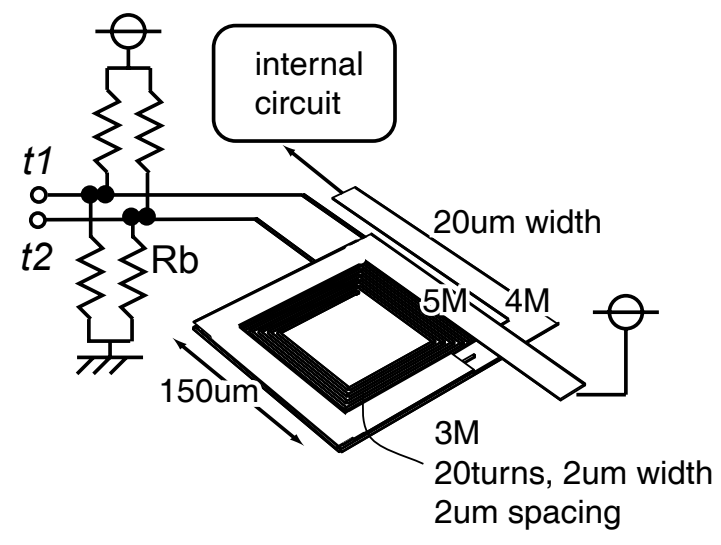

Figure 3: Mutual inductor structure.

The analog output current of the multiplier is proportional to $\left(V_{i n 1}-V_{i n 2}\right) \times\left(V_{i n 3}-V_{i n 4}\right)$. Here, in 1 and $i n 3$ in Fig. 4 are connected to $t 1$ in Fig. 3 , and $i n 2$, in $4, t 2$ are connected so that the output signal is proportional to the $d i / d t$ noise power.

The resistor of the low pass filter is formed using gatepoly without silicide and the resistance is about $100 \mathrm{k} \Omega$, the capacitor is formed using MIM capacitor and the capacitance is about $2.5 \mathrm{pF}$.

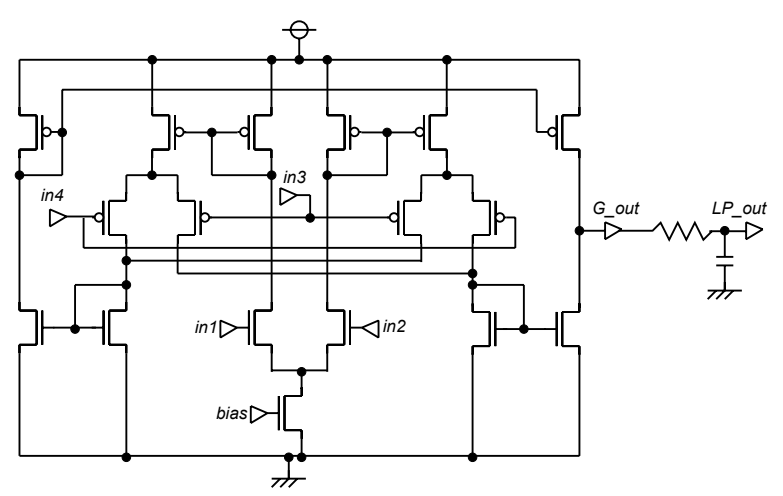

Figure 4: Gilbert multiplier and low pass filter.

\subsection{Comparators and Operation Mode Con- troller}

The comparator compares the $d i / d t$ noise power voltage with the reference voltage, and outputs " $\mathrm{H}$ " if the $d i / d t$ noise power voltage is higher than the reference voltage. We use two comparators and the reference voltages are $V_{\text {ref } L}$ and $V_{\text {refH }}$.

The schematic of the operation mode controller is shown in Fig.5. When the $d i / d t$ noise power is lower than $V_{\text {ref } L}$ (in $L=i n H=$ "L"), the operation mode controller outputs "H(all)" to the allORhal $f$ node so that all the internal circuit is turned on. When the $d i / d t$ noise power is higher than the threshold $V_{\text {refH }}(i n L=i n H=$ "H"), the operation mode controller outputs "L(half)" to the allORhalf node so that the half of the internal circuit is turned off and the $d i / d t$ is reduced. When the $d i / d t$ noise power is between $V_{\text {ref } H}$ and $V_{\text {refL }}$, the operation mode controller outputs the signal so as to keep the previous operation mode. 


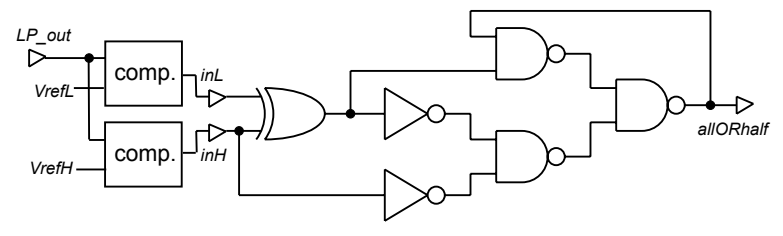

Figure 5: Schematic of the operation mode controller.

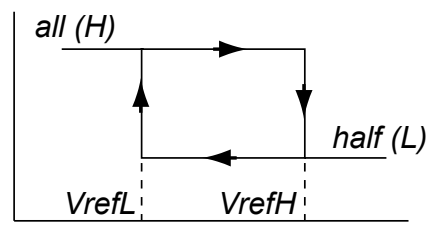

$\mathrm{di} / \mathrm{dt}$ noise power

Figure 6: Operation of the hysteresis comparator.

Thus, the comparators and the operation mode controller consist of a hysteresis comparator, as shown in Fig.6.

\section{Measurement}

\subsection{Setups}

The chip is designed and fabricated using $0.15 \mu \mathrm{m} \mathrm{5-}$ ML SOI-CMOS technology, and the chip area is about $3.2 \mathrm{~mm} \times 1.8 \mathrm{~mm}$, as shown in Fig.7.

The chip is mounted on a $\mathrm{Cu}$ board as shown in Fig.8. All the inputs are DC and supplied through lead lines to the "islands" on the board. The voltage of the islands are stabilized by several chip capacitors. $50 \Omega$ transmission lines are directly connected to the high-speed output pins including $C L K / 2, C L K / 32$. The allORhal $f$ signal is probed by a high impedance $(1 \mathrm{M} \Omega)$ probe.

\subsection{Frequency Dependence of $\mathrm{di} / \mathrm{dt}$ Noise Power}

When the same reference voltage is applied to $V_{\text {ref } H}$ and $V_{\text {refL }}$, and sweeping the voltage, the allORhalf signal changes when the reference voltage crosses the low pass filter output voltage which reflects the $d i / d t$ noise power. (Actually allORhal $f$ oscillates at a range of the reference voltage, which will be described in the next section, the center voltage is used here as the low pass filter output voltage, and hence the $d i / d t$ noise power.)

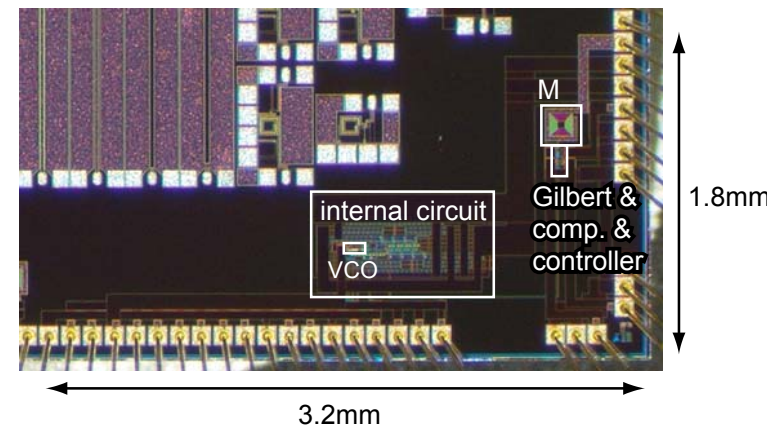

Figure 7: Chip photograph.

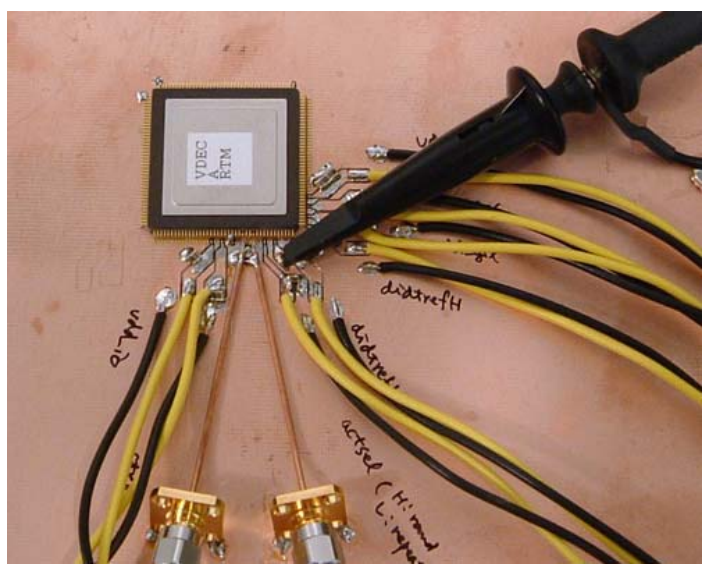

Figure 8: Measurement Setup.

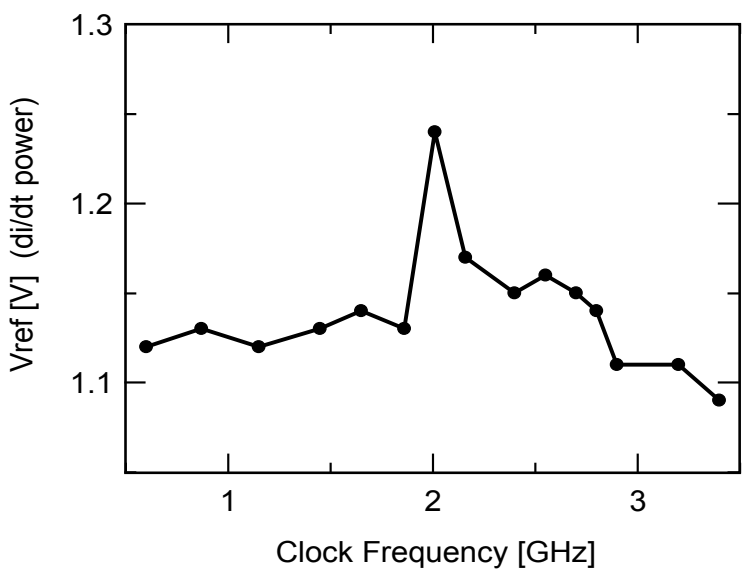

Figure 9: Clock frequency dependence of the di/dt noise power.

The clock frequency dependence of the $d i / d t$ noise power under $1.5 \mathrm{~V}$ power supply is shown in Fig.9. It shows that the $d i / d t$ noise power has a peak at around $2.0 \mathrm{GHz}$, which reflects the package and the bonding wire characteristics.

\section{Discussions}

\subsection{Waveforms of Oscillation}

As being described, the internal circuit has two modes: the all-active and half-active modes. If the $d i / d t$ noise power of the all-active is higher than $V_{r e f H}$ and the $d i / d t$ noise power of the half-active is lower than $V_{\text {ref } L}$, the internal circuit oscillates between the two modes. The H/L ratio depends on the average of the reference voltages $\left(V_{\text {ref } L}+\right.$ $\left.V_{\text {refH }}\right) / 2$, and the higher reference voltages lead higher $\mathrm{H}$ ratio. The oscillation frequency depends on the reference voltage difference $V_{\text {refH }}-V_{\text {ref } L}$ (and the low pass filter time constant), and the bigger reference voltage difference leads longer oscillation period.

The oscillation waveforms of allORhal $f$ signal between the all-active $(\mathrm{H})$ and the half-active $(\mathrm{L})$ modes are shown in Fig.10. The clock frequency is set to $2.0 \mathrm{GHz}$ in this measurement where the maximum $d i / d t$ is obtained as shown in Fig.9. 


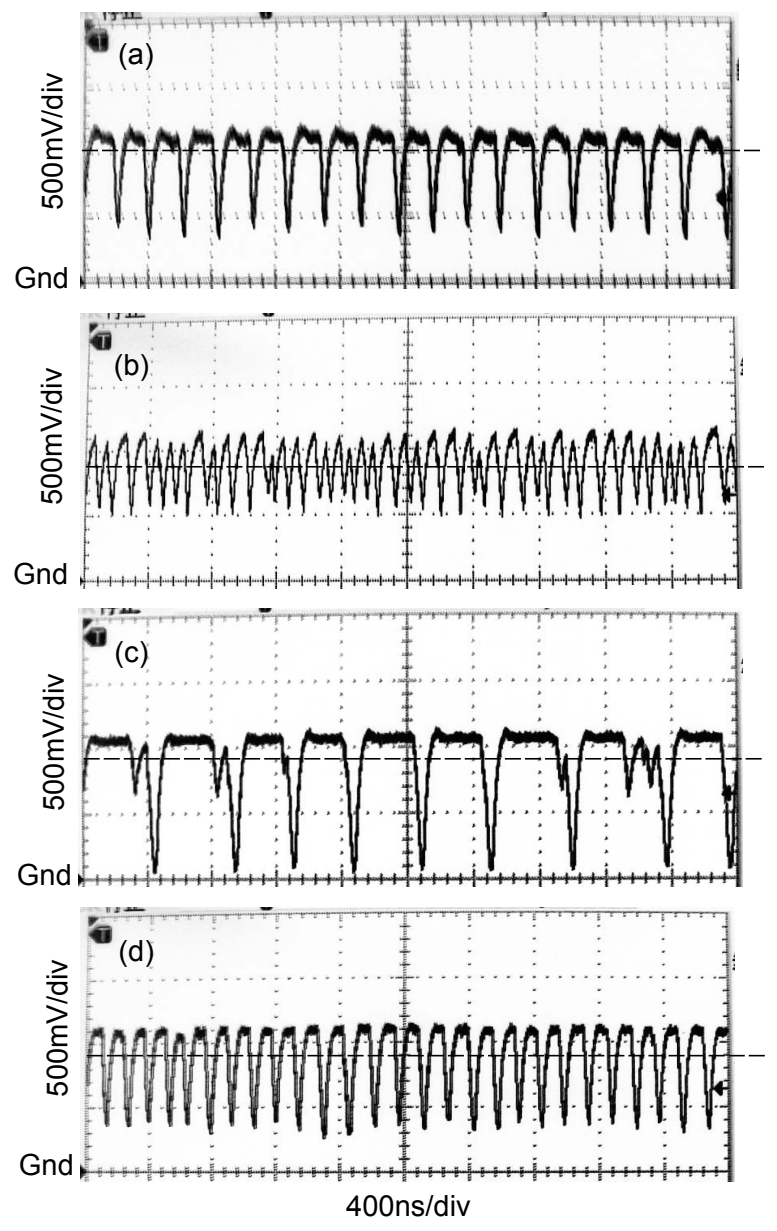

Figure 10: Measured waveforms of allORhal $f$ signal at 2.0 GHz clock frequency. The applied reference voltages, the measured results are summarized in Table 1.

\begin{tabular}{|r|c|c|r|r|}
\hline & $V_{\text {ref }}$ avrg.[V] & $\Delta V_{\text {ref }}[\mathrm{V}]$ & Out[V] & period[ns] \\
\hline \hline (a) & 1.275 & 0.01 & 1.0 & 222 \\
\hline (b) & 1.270 & 0.00 & 0.9 & 91 \\
\hline (c) & 1.270 & 0.02 & 0.9 & 444 \\
\hline (d) & 1.265 & 0.01 & 0.9 & 148 \\
\hline & 1.250 & 1.28 & L & - \\
\hline & 1.260 & 1.29 & $\mathrm{H}$ & - \\
\hline
\end{tabular}

Table 1: Reference voltage dependence of the oscillation period when the clock frequency is $2.0 \mathrm{GHz}$.

The applied reference voltages, the measured $\mathrm{H} / \mathrm{L}$ ratio and the oscillation period of (a), (b), (c) and (d) cases of Fig. 10 are summarized in Table 1 . Since the output driver of the allORhal $f$ signal is so small that the average output voltage of the waveforms is used here as the indication of the $\mathrm{H} / \mathrm{L}$ ratio.

For the H/L ratio, though the difference of case (b) and (c) is not clear because the $\Delta V_{\text {ref }}$ is not the same, the difference between (a) and (d) is clear and reasonable. For the oscillation period, it is clear that the oscillation period becomes longer as $\Delta V_{\text {ref }}$ becomes larger.
No oscillation occurs and stays "L" when $V_{\text {ref } L} \leq 1.25 \mathrm{~V}$ and stays "H" when $V_{\text {ref } H} \geq 1.29 \mathrm{~V}$ because the all-active has the $d i / d t$ noise power of $1.29-\delta[\mathrm{V}]$ and half-active has the $d i / d t$ noise power of $1.25+\delta[\mathrm{V}]$ where $0<\delta<0.01 \mathrm{~V}$.

\section{Conclusions}

An autonomous and margin aware $d i / d t$ noise control scheme has been demonstrated. A $d i / d t$ on the power line is detected by a mutual inductor, the induced voltage is squared by the Gilbert multiplier and the following low pass filter outputs the DC voltage in proportion to the $d i / d t$ noise power. The DC voltage is compared with reference voltages, and the mode of the internal circuit is controlled depending on the comparators output.

Though we used only two operation modes in this study. it can be easily extended to multiple operation modes. Our experimental results show that the mode of the internal circuit oscillates among the different operation modes so that the average $d i / d t$ noise power falls within a region determined by the reference voltages. It proves that our autonomous $d i / d t$ noise control scheme works as being designed.

This is the first demonstration, as the authors knowledge, of an autonomous and margin aware $d i / d t$ noise control scheme.

\section{Acknowledgments}

The VLSI chip in this study has been fabricated in the chip fabrication program of VLSI Design and Education Center(VDEC), the University of Tokyo in collaboration with OKI Corporation. This study was supported by Grant-inAid for JSPS Fellows of the Ministry of Education, Culture, Science and Technology.

\section{References:}

[1] Makoto Takamiya, Masayuki Mizuno, Kazuyuki Nakamura, "An on-chip 100GHz-sampling rate 8channel sampling oscilloscope with embedded sampling clock generator," IEEE Int. Solid-State Circuit Conf., pp.182-183, Feb. 2002.

[2] Sani Nassif "Delay Variability: Sources, Impacts and Trends," IEEE Int. Solid-State Circuit Conf., WP 224, Feb. 2000.

[3] Toru Nakura, Makoto Ikeda, Kunihiro Asada, "Power Supply di/dt Measurement using On-chip di/dt Detector Circuit," IEEE/JSAP Symposium on VLSI Circuits, pp.106-109, June 2004.

[4] Barrie Gilbert, "A Precise Four-Quadrant Multiplier with Subnanosecond Response," IEEE J. Solid-State Circuits, pp.365-373, Dec. 1968. 